\title{
Multifrequency and Multistatic Inverse Synthetic Aperture Radar, with Application to FM Passive Radar
}

\author{
Guillaume Ginolhac, Françoise Schmitt, Franck Daout, and Philippe Forster
}

SATIE, ENS CACHAN, Université Paris Sud, 61 avenue du President Wilson, 94235 Cachan, France

Correspondence should be addressed to Guillaume Ginolhac, guillaume.ginolhac@satie.ens-cachan.fr

Received 27 April 2009; Accepted 20 October 2009

Academic Editor: Carlos Lopez-Martinez

Copyright ( $) 2010$ Guillaume Ginolhac et al. This is an open access article distributed under the Creative Commons Attribution License, which permits unrestricted use, distribution, and reproduction in any medium, provided the original work is properly cited.

\begin{abstract}
This paper deals with the imaging of a moving target using a multifrequency and multistatic radar consisting in one receiver and several narrowband transmitters. Considering two hypotheses about the studied target, we derive two multistatic inverse synthetic aperture radar processors: the first one, which models the target as a set of isotropic points, performs a coherent sum of bistatic images; the second one, which models the target as a set of nonisotropic points, performs an incoherent sum of bistatic images. Numerical simulations are done, which demonstrate the efficiency of the second processor. We also apply both processors to a multistatic passive radar scenario for which the transmitters are FM stations located in a realistic configuration. We study the system performance in terms of resolution and sidelobe levels as a function of the number of transmitters and of the integration time. Both processors are applied to similar complex targets for which the scattered fields are simulated by a numerical electromagnetic code. The resulting multistatic radar images show interesting characteristics that might be used by classification algorithms in future work.
\end{abstract}

\section{Introduction}

Passive radars use one or several transmitters of opportunity to detect, locate, and classify targets. This technique is cost effective because users only need to install receivers. Nevertheless, the use of civil communication signals, which are often narrowband signals, makes the detection and the classification difficult. The main limitations of passive radars are due to the center frequency and the bandwidth of these signals. For instance, for FM and TV signals, the center frequency varies from $100 \mathrm{MHz}$ (for FM) to $800 \mathrm{MHz}$ (for some TV stations in France) with bandwidths ranging from $75 \mathrm{kHz}$ (for $\mathrm{FM}$ ) to $1 \mathrm{MHz}$ (for TV stations). For comparison, the center frequency and the bandwidth of a monostatic active radar such as the RAMSES ONERA SAR system [1] in the X-band are approximately $10 \mathrm{GHz}$ and 1.2 GHz. For reaching equivalent performances (resolution and sidelobe levels) as an active system, a passive radar has to illuminate the target a long time and to consider several narrowband transmitters (FM or TV) in order to compensate for the low values of the center frequency and the bandwidth. In the following, such a radar system will be called a multifrequency and multistatic radar.

Passive bistatic radar systems consisting in one transmitter and one receiver located at different places and using FM [2] and TV signals [3] have demonstrated the feasibility of passive radars. Detection, localization, and tracking algorithms have been developed and validated using real data. However, classification is better performed using multiple transmitters or receivers. Therefore, different approaches have recently been developed. An approach to Automatic Target Recognition (ATR) consists in comparing the modeled Radar Cross Section (RCS) of different targets with those measured by the multistatic radar system [47]. Another approach, which is the one this paper focuses on, relies on the development of classification algorithms based on a target image obtained from the multistatic radar measurements.

In previous works $[8,9]$, we studied the resolution attainable by a multifrequency and multistatic radar using 


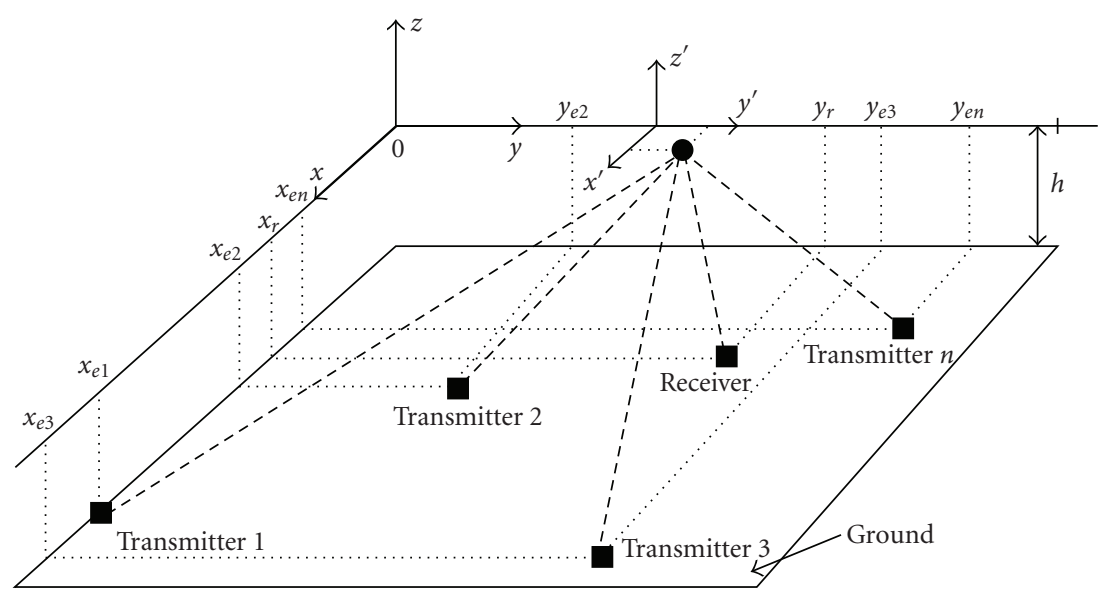

FIgURE 1: General scheme of a multifrequency and multistatic radar.

analytical and numerical approaches. Results showed that multifrequency and multistatic radars could achieve sufficient resolution using several narrowband transmitters and a long integration time. In this paper, we propose imaging algorithms for multifrequency and multistatic radars, which could be useful as a preprocessing for classification methods. Most of imaging algorithms are based on the Synthetic Aperture Radar (SAR) concept [10]. The main purpose of imaging algorithms is to achieve enough resolution to make the classification step easy. For instance, the final images corresponding to two similar targets must exhibit enough differences so that it is possible to make the difference. For narrowband radar, it is well known that data from transmitter-receiver pairs distributed all around the target allow to have an image $[11,12]$ with such properties. These particular systems are similar to tomographic ones. Recently, $\mathrm{Wu}$ and Munson [13] simulated the imaging of a moving airplane using a multistatic radar composed of several TV transmitters and a single receiver. The imaging algorithm in [13] relies on the assumption that the target is composed of isotropic points and uses $2 \mathrm{D}$ interpolation on the frequency domain to achieve the processing. However, the resulting images are subject to degradation because the target has to be illuminated over a long period (in this case, the target is often nonstationary). Several methods have been proposed for solving this problem: time-frequencybased methods [14-16], deconvolution methods [17], or optimization techniques [18].

This paper proposes new Multistatic Inverse SAR (MISAR) processors where SAR processors were showed to be particular cases of detection algorithms $[19,20]$. Two MISAR processors are developed, depending on the hypotheses made about the target: in the first case the target is assumed to be equivalent to a set of isotropic points, and in the second case the target is assumed to be equivalent to a set of nonisotropic points. We demonstrate that the first processor is equivalent to the one proposed in [13] while the second one is slightly different.

Next, we apply both MISAR processors to a realistic multistatic FM passive radar configuration for which the
FM transmitter locations are chosen according to those of the FM transmitters in France [21]. A study of image resolution based on methods presented in $[8,9]$ is performed as a function of the number of transmitters and of the integration time. Both MISAR processors are tested on two similar complex targets presented in [22]. This paper does not consider the estimation of the trajectory. This problem is addressed with an extended Kalman Filter or a particle filter in $[2,3,7,23-26]$.

Section 2 describes the hypotheses that are made about the target as well as the notations that will be used in this paper. Section 3 develops both MISAR processors and illustrates their potential via a numerical simulation. Section 4 details the geometric configuration of our multistatic passive FM radar scenario and presents the resulting image.

\section{Problem Statement}

We first present the notation needed to derive the desired detection algorithms. We next present two conjectures: the scattered field of target is either a set of isotropic points or a set of nonisotropic points. The corresponding detection problems are finally presented.

2.1. Context and Notation. Consider $N$ narrowband transmitters (FM, TV, ...), each of them transmitting a continuous signal $\mathfrak{R}\left(e_{n}(t) \exp \left(j 2 \pi f_{0}(n) t\right)\right)$ where $n$ denotes the transmitter index $(1 \leq n \leq N) . f_{0}(n)$ is the center frequency of the transmitted signal and $e_{n}(t)$ is the baseband signal. Let $\left(x_{e n}, y_{e n}\right)_{1 \leq n \leq N}$ be the cartesian coordinates of the transmitters as shown in Figure 1. The receiver, which is located at position $\left(x_{r}, y_{r}\right)$, collects the reflected signals and performs $N$ complex demodulations with respect to $f_{0}(n)$. The direct path signals from each transmitter are assumed to have been removed by appropriate processing. The sampling period is denoted by $T_{s}$, and the receiver collects $N_{s}$ samples from each transmitter. Signals $e_{n}$ are assumed normalized according to $\sum_{k=0}^{N_{s}-1}\left|e_{n}\left(k T_{s}\right)\right|^{2}=1$. It is also assumed that only a single target is present, flying along a known trajectory which is considered to be along the $y$-axis 
at height $h$ with a constant velocity $v$. We consider a receiver such that its aperture enables the target detection located between $y_{\min }$ and $y_{\max }$. A moving coordinate system $\mathcal{R}^{\prime}$, with origin $O^{\prime}\left(0, y_{k}, 0\right)$, is used for the target point coordinates $\left(x^{\prime}, y^{\prime}, z^{\prime}\right)$, where $y_{k}=y_{\min }+k T_{s} v$. For clarity, only $2 \mathrm{D}$ imagery is considered.

2.2. Target Hypotheses. In this paper, we consider narrowband transmitters. As a consequence, the scattering function of the general target, which is denoted by $\gamma_{t}$, can be assumed to be independent of the transmitted frequency. Moreover, assuming that the target can be described as a set of points,

$$
\gamma_{t}=\sum_{i=1}^{N_{p}} \gamma_{i}\left(\theta_{i t}, \phi_{i t}, \theta_{i r}, \phi_{i r}\right) \delta\left(x^{\prime}-x_{i}^{\prime}, y^{\prime}-y_{i}^{\prime}\right),
$$

where $N_{p}$ is the number of scattering points of the target, $\quad\left(x_{i}^{\prime}, y_{i}^{\prime}\right)_{1 \leq i \leq N_{p}}$ are the scalar coordinates of these points, and $\left(\theta_{i t}, \phi_{i t}, \theta_{i r}, \phi_{i r}\right)$ are the incident and observed angles defined in Figure 2. Since angles $\left(\theta_{i t}\left(k T_{s}, n\right), \phi_{i t}\left(k T_{s}, n\right), \theta_{i r}\left(k T_{s}\right), \phi_{i r}\left(k T_{s}\right)\right)$ vary as a function of the target position and the transmitter number (indexed by $n$ ), the scattering coefficient $\gamma_{i}$ is, without additional assumptions, a function of $k T_{s}$ (target position) and $n$ (transmitter number). In this paper with a view to develop our MISAR processors, the scattering coefficient $\gamma_{i}$ is assumed to be independent of $k T_{s}$.

Given this initial hypothesis, we consider two cases:

(i) the isotropic case for which the scattering coefficient $\gamma_{i}$ neither depends on the transmitter nor depends on the receiver location; in this case, the target can be considered to be a set of isotropic points;

(ii) the nonisotropic case for which the scattering coefficient $\gamma_{i}$ depends on the transmitter and receiver locations (indexed by $n$ ); in this case, the $\gamma_{\text {in }}$ coefficients of each transmitter $n$ are different and the target is considered as a set of nonisotropic points.

In the following, we denote by $\gamma$ the scattered field of the isotropic point located at pixel $\left(x^{\prime}, y^{\prime}\right)$ and by $\gamma_{n}$ the scattered field seen by transmitter $n$ of the nonisotropic point located at pixel $\left(x^{\prime}, y^{\prime}\right)$.

2.3. Detection Problem for the Isotropic Case. We consider the following detection problem for the pixel at $\left(x^{\prime}, y^{\prime}\right)$; the received signal may be composed either of noise (hypothesis $H_{0}$ ) or of noise plus the reflected target signal (hypothesis $\left.H_{1}\right)$ :

$$
\begin{aligned}
& H_{0}: z_{n}\left(k T_{s}\right)=b_{n}\left(k T_{s}\right), \\
& H_{1}: z_{n}\left(k T_{s}\right)=\gamma s_{n}\left(k T_{s}, x^{\prime}, y^{\prime}\right)+b_{n}\left(k T_{s}\right),
\end{aligned} \quad \forall n,
$$

where $z_{n}\left(k T_{s}\right)$ is the signal received from transmitter $n$, $b_{n}\left(k T_{s}\right)$ is complex zero mean Gaussian noise with known variance $\sigma^{2}$, and $s_{n}$ is the noiseless signal reflected by a

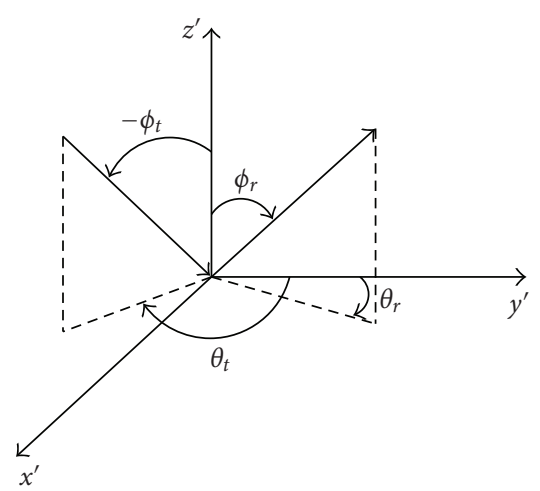

FIgure 2: Definitions of incident and observed angles.

normalized isotropic point, originating from the transmitter $n$. The expression for $s_{n}$ is

$$
\begin{aligned}
s_{n}\left(k T_{s}, x^{\prime}, y^{\prime}\right)= & e_{n}\left(k T_{s}-\tau_{n}\left(k, x^{\prime}, y^{\prime}\right)\right) \\
& \times \exp \left(-2 i \pi f_{0}(n) \tau_{n}\left(k, x^{\prime}, y^{\prime}\right)\right), \\
\tau_{n}\left(k, x^{\prime}, y^{\prime}\right)= & \frac{1}{c}\left(\sqrt{\left(x_{e n}-x^{\prime}\right)^{2}+\left(y_{k}+y^{\prime}-y_{e n}\right)^{2}+h^{2}}\right. \\
& \left.+\sqrt{\left(x_{r}-x^{\prime}\right)^{2}+\left(y_{k}+y^{\prime}-y_{r}\right)^{2}+h^{2}}\right),
\end{aligned}
$$

where $\tau_{n}$ is the time delay along the transmitter $n$-targetreceiver path. The scattering coefficient $\gamma$ is unknown.

Define the vectors $\mathbf{z}_{n}=\left[z_{n}(0) \cdots z_{n}\left(\left(N_{s}-1\right) T_{s}\right)\right]^{T}$, $\mathbf{s}_{n}\left(x^{\prime}, y^{\prime}\right)=\left[s_{n}\left(0, x^{\prime}, y^{\prime}\right) \cdots s_{n}\left(\left(N_{s}-1\right) T_{s}, x^{\prime}, y^{\prime}\right)\right]^{T}, \mathbf{b}_{n}=$ $\left[b_{n}(0) \cdots b_{n}\left(\left(N_{s}-1\right) T_{s}\right)\right]^{T}$ (where ${ }^{T}$ is the symbol for the transpose operation), and

$$
\begin{aligned}
& \mathbf{z}=\left[\mathbf{z}_{1}^{T} \cdots \mathbf{z}_{N}^{T}\right]^{T}, \\
& \mathbf{d}\left(x^{\prime}, y^{\prime}\right)=\left[\mathbf{s}_{1}^{T}\left(x^{\prime}, y^{\prime}\right) \cdots \mathbf{s}_{N}^{T}\left(x^{\prime}, y^{\prime}\right)\right]^{T}, \\
& \mathbf{d}\left(x^{\prime}, y^{\prime}, \gamma\right)=\gamma \mathbf{d}\left(x^{\prime}, y^{\prime}\right), \\
& \mathbf{b}=\left[\mathbf{b}_{1}^{T} \cdots \mathbf{b}_{N}^{T}\right]^{T} .
\end{aligned}
$$

The initial detection problem (2) may be rewritten as

$$
\begin{aligned}
& H_{0}: \mathbf{z}=\mathbf{b}, \\
& H_{1}: \mathbf{z}=\mathbf{d}\left(x^{\prime}, y^{\prime}, \gamma\right)+\mathbf{b},
\end{aligned}
$$

where $\gamma$ is unknown.

2.4. Detection Problem for the Nonisotropic Case. We consider the following detection problem for the pixel at $\left(x^{\prime}, y^{\prime}\right)$, where the received signal may be composed either of noise (hypothesis $H_{0}$ ) or of noise plus the reflected target signals (hypothesis $H_{1}$ ):

$$
\begin{aligned}
& H_{0}: z_{n}\left(k T_{s}\right)=b_{n}\left(k T_{s}\right), \\
& H_{1}: z_{n}\left(k T_{s}\right)=\gamma_{n} s_{n}\left(k T_{s}, x^{\prime}, y^{\prime}\right)+b_{n}\left(k T_{s}\right),
\end{aligned} \quad \forall n,
$$




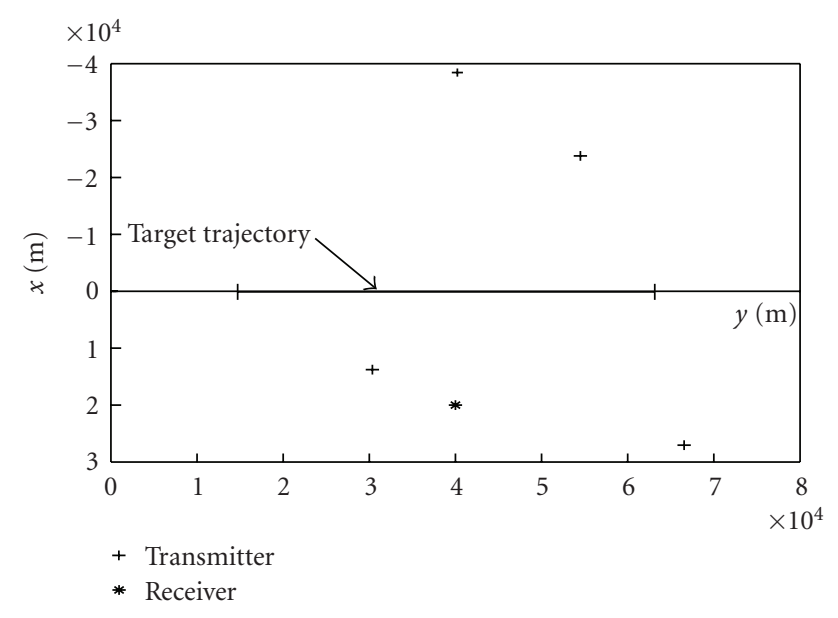

FIGURE 3: Transmitters and receiver locations and target trajectory.

where the definitions of $z_{n}\left(k T_{s}\right), b_{n}\left(k T_{s}\right)$, and $s_{n}$ are the same as those of Section 2.3. The scattering coefficients $\gamma_{n}$ are unknown.

For the nonisotropic case, $\mathbf{d}$ is defined as follows:

$$
\mathbf{d}\left(x^{\prime}, y^{\prime}, \gamma_{1}, \ldots, \gamma_{N}\right)=\left[\gamma_{1} \mathbf{s}_{1}^{T}\left(x^{\prime}, y^{\prime}\right) \cdots \gamma_{N} \mathbf{s}_{N}^{T}\left(x^{\prime}, y^{\prime}\right)\right]^{T} .
$$

The initial detection problem (6) may be rewritten as

$$
\begin{aligned}
& H_{0}: \mathbf{z}=\mathbf{b}, \\
& H_{1}: \mathbf{z}=\mathbf{d}\left(x^{\prime}, y^{\prime}, \gamma_{1}, \ldots, \gamma_{N}\right)+\mathbf{b},
\end{aligned}
$$

where $\left(\gamma_{1}, \ldots, \gamma_{N}\right)$ are unknown.

\section{Multistatic ISAR Processors}

Both the isotropic (5) and the nonisotropic (8) detection problems described above are addressed in Sections 3.1 and 3.2. Moreover, simulations of the two resulting MISAR processors are provided and analyzed in Section 3.3.

3.1. MISAR Processor for Isotropic Case. The Generalized Likelihood Ratio Test for the detection problem (5) leads to

$$
\frac{\max _{\gamma} f_{\mathbf{z}} / H_{1}}{f_{\mathbf{z}} / H_{0}} \underset{H_{0}}{\gtrless} \eta,
$$

where $f_{\mathbf{z}} / H_{i}$ is the probability density function of vector $\mathbf{z}$ under the hypothesis $H_{i}$. The threshold $\eta$ is usually adjusted to fulfill a given probability of false alarm. In Gaussian context, the above test may be rewritten as

$$
\max _{\gamma}\|\mathbf{z}\|^{2}-\left\|\mathbf{z}-\mathbf{d}\left(x^{\prime}, y^{\prime}, \gamma\right)\right\|^{2} \underset{H_{0}}{\stackrel{H_{1}}{\gtrless}} \eta^{\prime} .
$$

Estimation of $\gamma$ consists in determining

$$
\underset{\gamma}{\operatorname{argmin}}\left\|\mathbf{z}-\mathbf{d}\left(x^{\prime}, y^{\prime}, \gamma\right)\right\|^{2},
$$

which leads to the least-squares solution

$$
\hat{\gamma}=\mathbf{d}^{*}\left(x^{\prime}, y^{\prime}\right) \mathbf{z}
$$

where $*$ denotes the conjugate transpose operation. Substituting $\hat{\gamma}$ into (10),

$$
\left|\sum_{n=1}^{N} \mathbf{s}_{n}^{*}\left(x^{\prime}, y^{\prime}\right) \mathbf{z}_{n}\right|^{2} \stackrel{H_{H_{0}}}{\gtrless} \eta .
$$

We propose to compose the target image $I$ by plotting the test value of (13) for every $\left(x^{\prime}, y^{\prime}\right)$ position:

$$
I\left(x^{\prime}, y^{\prime}\right)=\left|\sum_{n=1}^{N} \mathbf{s}_{n}^{*}\left(x^{\prime}, y^{\prime}\right) \mathbf{z}_{n}\right|^{2} .
$$

Let $I_{n}\left(x^{\prime}, y^{\prime}\right)=\mathbf{s}_{n}^{*}\left(x^{\prime}, y^{\prime}\right) \mathbf{z}_{n}$ denote the bistatic complex image created using only the transmitter $n$. It is to be noted that (14) makes a coherent sum of every bistatic complex image which is identical to classic multistatic algorithms [13]. We call this algorithm Multistatic Coherent ISAR (MCISAR) processing.

The computation cost of the bistatic complex image $I_{n}$ can prove to be heave. Nevertheless, an approximation such as the bistatic PFA proposed in [27] could be used with a view to better computational efficiency.

3.2. MISAR Processor for Nonisotropic Case. The Generalized Likelihood Ratio Test for the detection problem (8) leads to

$$
\frac{\max _{\gamma_{1}, \ldots, \gamma_{N}} f_{\mathbf{z}} / H_{1}}{f_{\mathbf{z}} / H_{0}} \underset{H_{0}}{\stackrel{H_{1}}{\gtrless}} \eta \text {. }
$$

In Gaussian context, the above test may be rewritten as

$$
\max _{\gamma_{1}, \ldots, \gamma_{N}}\|\mathbf{z}\|^{2}-\left\|\mathbf{z}-\mathbf{d}\left(x^{\prime}, y^{\prime}, \gamma_{1}, \ldots, \gamma_{N}\right)\right\|^{2} \underset{H_{0}}{\stackrel{H_{1}}{\gtrless}} \eta^{\prime} .
$$

Estimation of $\left(\gamma_{1}, \ldots, \gamma_{N}\right)$ consists in determining

$$
\underset{\gamma_{1}, \ldots, \gamma_{N}}{\operatorname{argmin}}\left\|\mathbf{z}-\mathbf{d}\left(x^{\prime}, y^{\prime}, \gamma_{1}, \ldots, \gamma_{N}\right)\right\|^{2},
$$

which leads to the least-squares solution

$$
\hat{\gamma}_{n}=\mathbf{s}_{n}^{*}\left(x^{\prime}, y^{\prime}\right) \mathbf{z}_{n} \quad \text { for } 1 \leq n \leq N \text {. }
$$

Substituting $\hat{\gamma}_{n}$ into (16),

$$
\sum_{n=1}^{N}\left|\mathbf{s}_{n}^{*}\left(x^{\prime}, y^{\prime}\right) \mathbf{z}_{n}\right|^{2} \underset{H_{0}}{\stackrel{H_{1}}{\gtrless}} \eta \text {. }
$$

We propose to compose target image $I$ by plotting the test value of (19) for every $\left(x^{\prime}, y^{\prime}\right)$ position:

$$
I\left(x^{\prime}, y^{\prime}\right)=\sum_{n=1}^{N}\left|\mathbf{s}_{n}^{*}\left(x^{\prime}, y^{\prime}\right) \mathbf{z}_{n}\right|^{2} .
$$




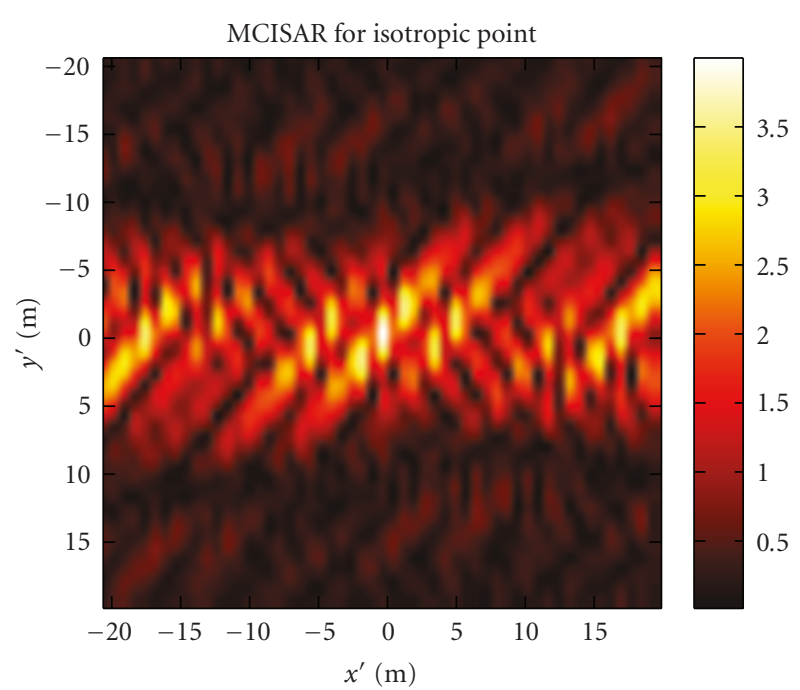

(a)

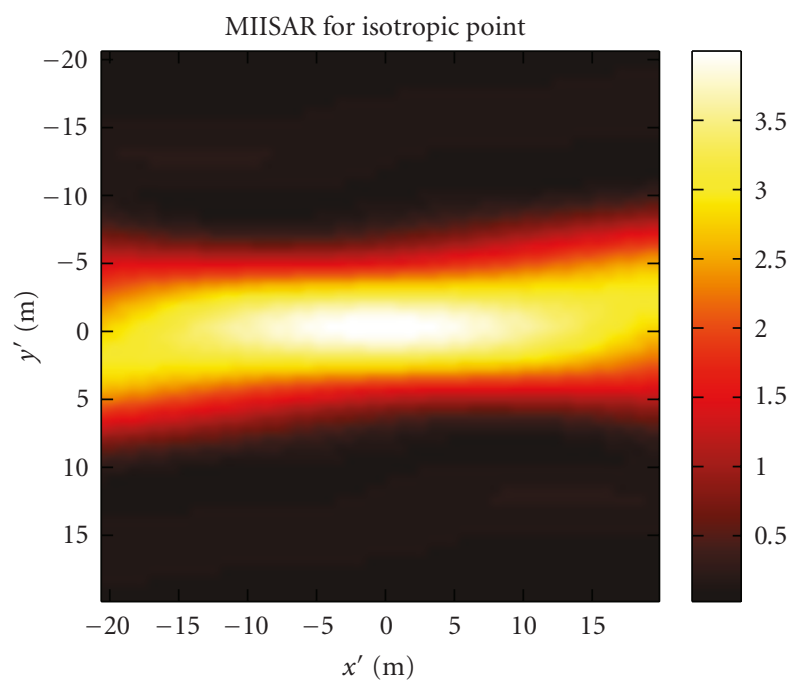

(c)

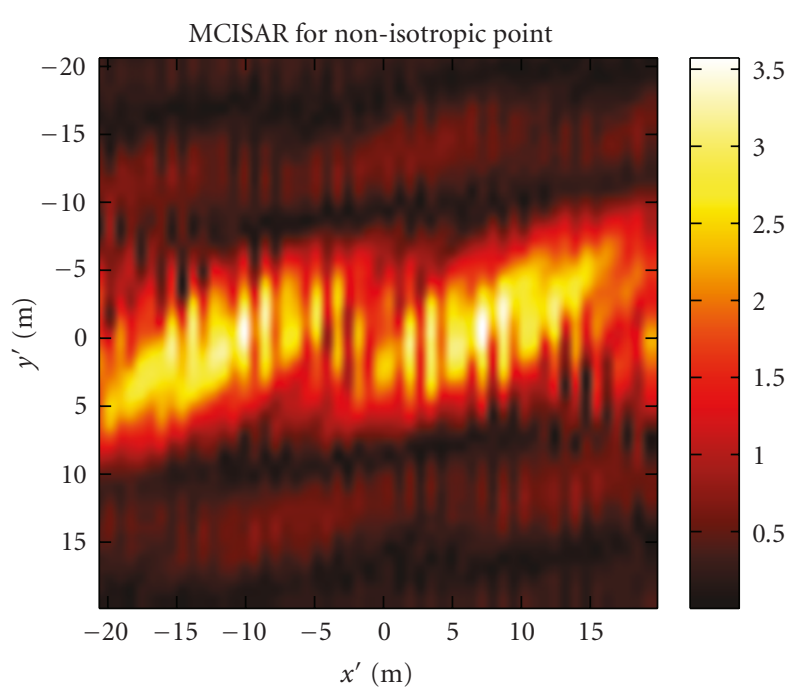

(b)

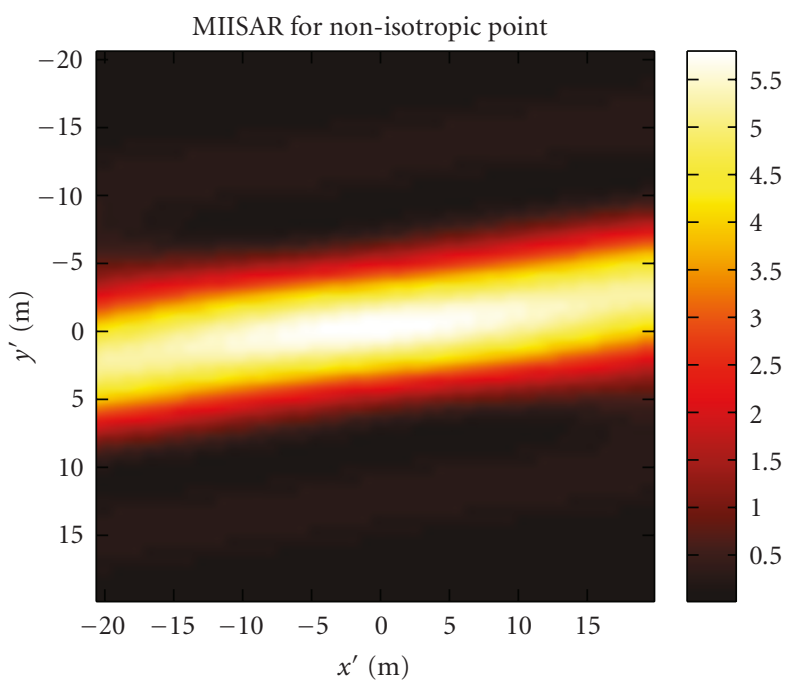

(d)

FIGURE 4: MCISAR result for an isotropic point (a) and nonisotropic point (b) and MIISAR result for an isotropic point (c) and nonisotropic point (d).

Equation (20) is an incoherent sum of every bistatic complex image. This result is consistent with the fact that the scattering coefficients are assumed to be different for each transmitter. Equation (20) thus differs from MCISAR (14) and classic multistatic algorithms that usually perform a coherent sum of every bistatic complex image. Such a result emphasizes our interest in modeling the received signal more accurately. We call this algorithm Multistatic Incoherent ISAR (MIISAR) processing.

\subsection{Qualitative Comparison of MCISAR and MIISAR. With} a view to compare the MCISAR and MIISAR processing, we perform two simulations. The first one considers the target as an isotropic point, and the second one as a nonisotropic point, both located at the center of the mobile reference $\left(x^{\prime}, y^{\prime}\right)=(0,0)$. The radar configuration, for which four FM transmitters are used, is given in Figure 1. The parameters of the simulation are summarized in Table 1 . The values of the scattering coefficients for the nonisotropic point have no particular physical meaning because they were randomly chosen. The target speed is $150 \mathrm{~m} / \mathrm{s}$. The synthetic array length is $5.25 \mathrm{~km}$ long, which corresponds to an integration time of 35 seconds. The receiver is located at $(40,20) \mathrm{km}$. The positions of the transmitters and receiver, as well as the target trajectory, are also shown in Figure 3.

Results of MCISAR and MIISAR considering both the isotropic and the nonisotropic points are displayed in Figure 4. Regarding the isotropic point, it is to be noted that the MIISAR result is the envelope of the MCISAR one. However, an important drawback of the MCISAR method should be pointed out for the nonisotropic point case: there are two principal lobes for only one point. On the other hand, the MIISAR result for the isotropic point is similar to that 
TABle 1: Parameters.

\begin{tabular}{lccll}
\hline Transmitter number & Center frequency $(\mathrm{MHz})$ & Location $(\mathrm{km})$ & $\begin{array}{l}\text { Scattering coefficient of } \\
\text { isotropic point }\end{array}$ & $\begin{array}{l}\text { Scattering coefficient of } \\
\text { nonisotropic point (modulus, } \\
\text { phase) }\end{array}$ \\
\hline 1 & 100 & $(-23.8,54.45)$ & 1 & $\left(0.66,-61.11^{\circ}\right)$ \\
2 & 100.2 & $(13.8,30.3)$ & 1 & $\left(2.19,85.54^{\circ}\right)$ \\
3 & 100.4 & $(27,66.5)$ & 1 & $\left(0.22,-144.16^{\circ}\right)$ \\
4 & 100.6 & $(-38.4,40.2)$ & 1 & $\left(0.73,-8.69^{\circ}\right)$ \\
\hline
\end{tabular}

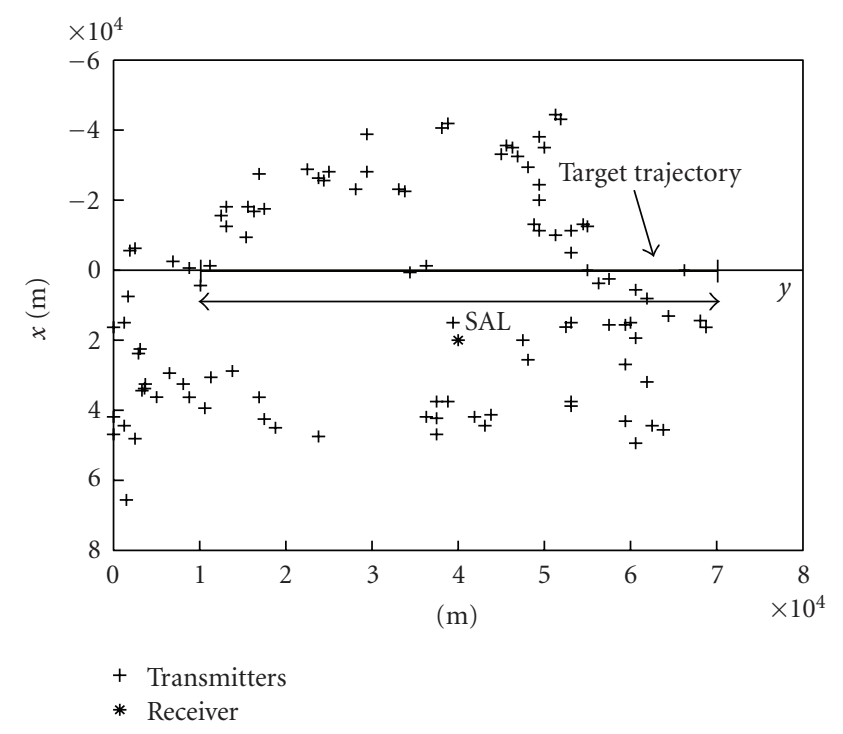

Figure 5: Positions of the 103 transmitters and of the receiver and of the target trajectory.

corresponding to the nonisotropic point. Such simulation results enable the use of MIISAR processing to be envisaged for target recognition.

In the next section a quantitative study of both MISAR processors is performed. Resolution and sidelobe levels are studied in a realistic ISAR context and the MCISAR and MIISAR processors are applied to complex targets.

\section{Simulations with FM Passive Multistatic Radar}

The first subsection describes the FM passive radar configuration and the considered targets. The second subsection is dedicated to the study of the impact of the system geometry on the image resolution for the MIISAR. Finally, in the third subsection, the MCISAR and MIISAR processors are applied to complex targets and the results are analyzed.

4.1. Simulation Context. As a realistic ISAR context configuration, which is shown in Figure 5, we chose transmitter locations corresponding to the FM transmitters that exist near Gueret (a small city in the center of France) where up to 103 transmitters are available in a $100 \mathrm{~km}$ side square [21]. For the sake of illustration, the transmitters are assumed to

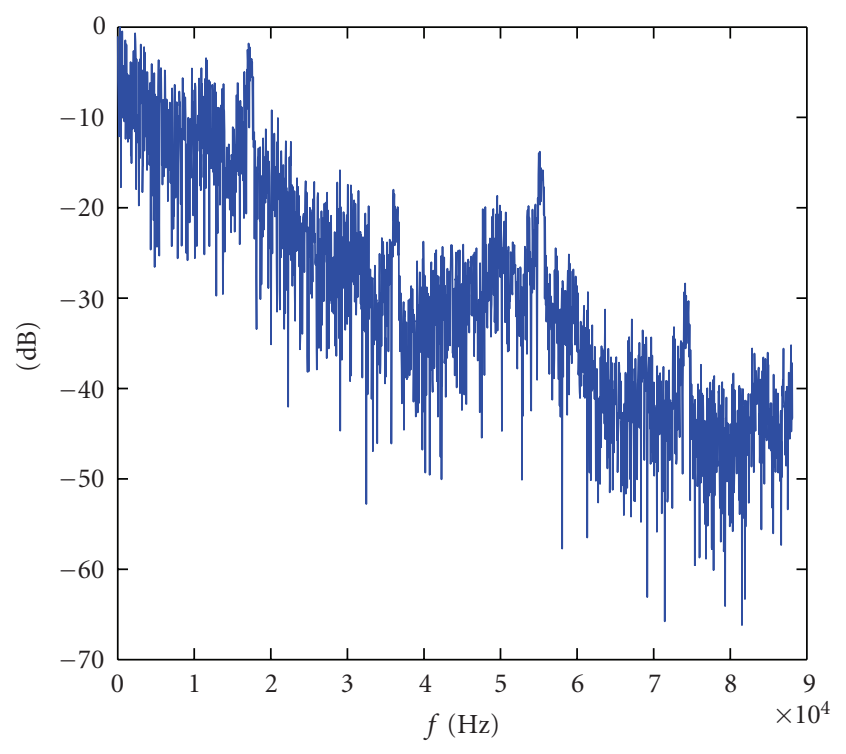

FIGURE 6: Example of baseband transmitted signal spectrum.

provide sufficient Signal-to-Noise Ratio (SNR) so that the target is visible to all of them. However, in practice such a case is unlikely to happen. The receiver is located at coordinates $(40,20) \mathrm{km}$ in the $(x, y)$ plane. The height of the target flight $h$ is 100 meters.

The center frequencies of each of the $N=103$ narrowband transmitters are all different and are separated by at least $200 \mathrm{kHz}$. We assume that the center frequency $f_{0}(n)$ for transmitter $n$ is equal to $100+(n-(N-1) / 2) 0.2 \mathrm{MHz}$. This choice was made so as to have the total frequency coverage in the FM band and therefore to obtain the best image resolution in the chosen geometric configuration.

The FM signal for each transmitter is assumed to carry "rock music" in order to cover a maximum bandwidth. The music signal is frequency modulated according to the commercial FM standard [24]. An example of the transmitted baseband signal spectrum normalized with respect to its maximum is shown in Figure 6. Notice that the signal power is not constant over the frequency band and that the bandwidth could be considered as quite small. The duration of the received signal depends on the Synthetic Array Length (SAL) and the speed of the target. The baseband signals are sampled at $F_{s}=170 \mathrm{kHz}$.

To study the impact of the geometric configuration of Figure 5 on resolution, Section 4.2 considers the target to be 


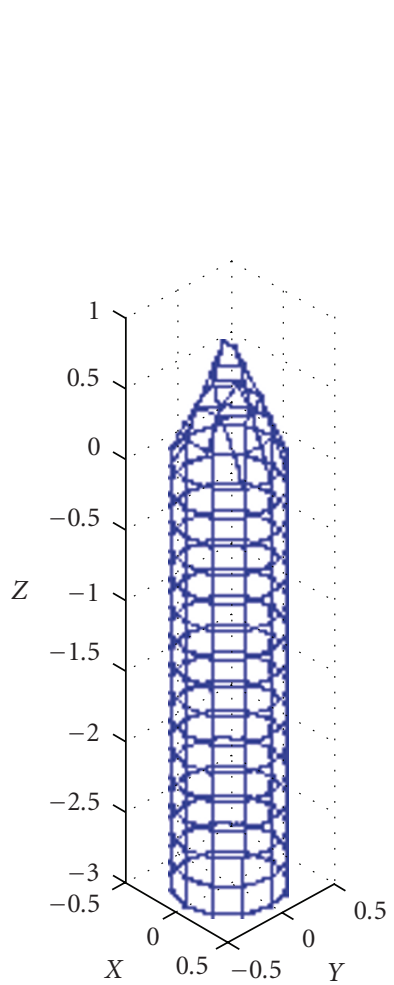

(a)

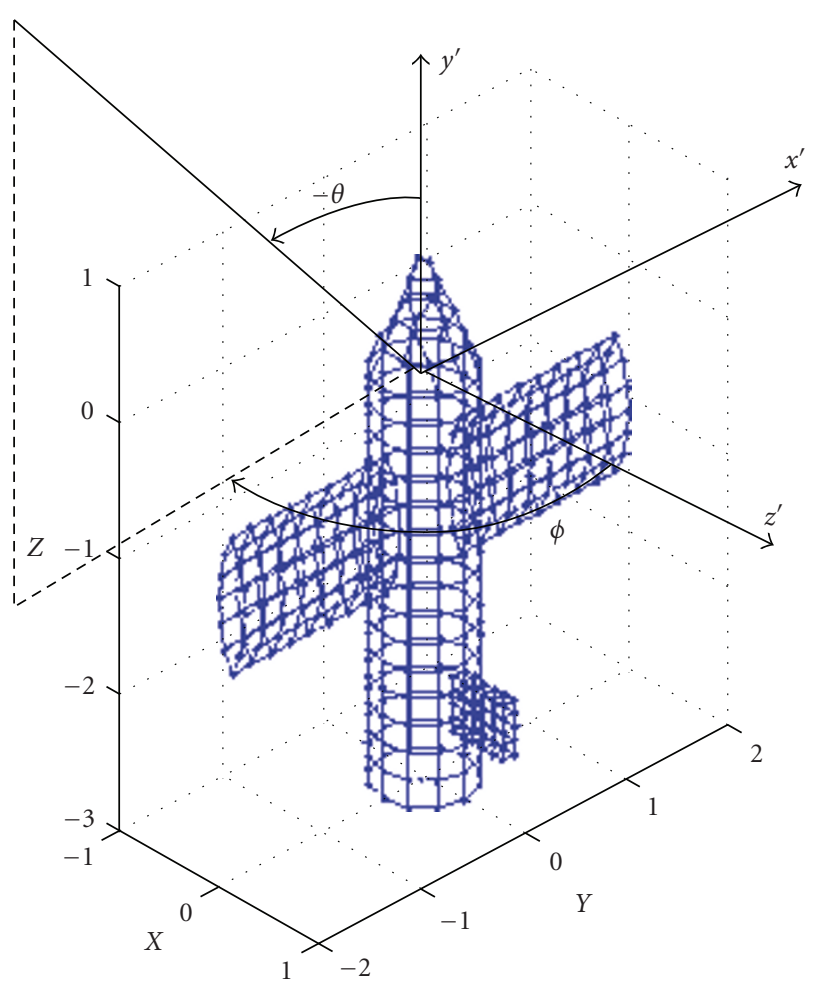

(b)

FIgURE 7: Targets "cycone" (a) and airplane (b).

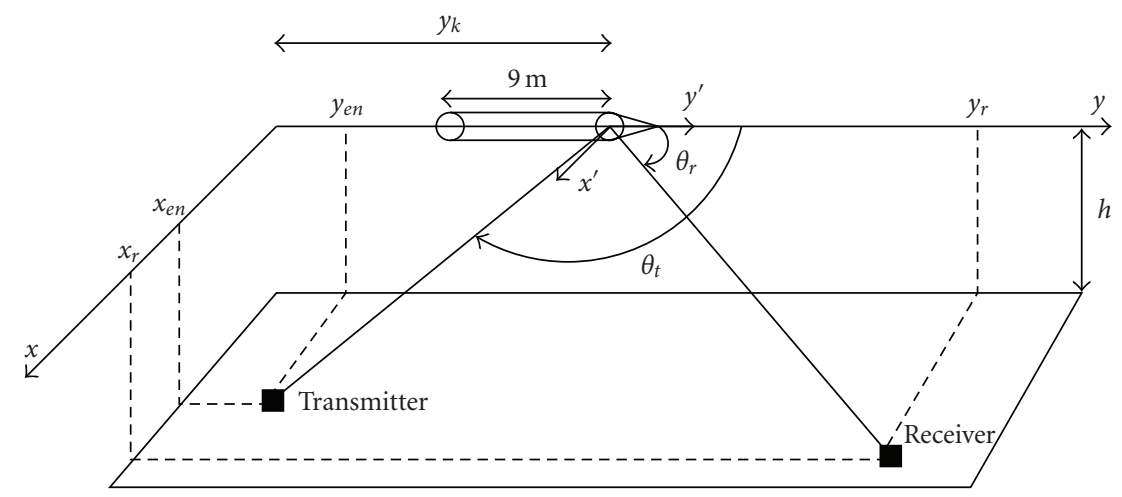

FIgURE 8: Target position in the moving coordinate system $\mathcal{R}^{\prime}$.

an isotropic point. Section 4.3 considers the two complex targets presented in [22]: a "cycone" and an airplane, shown in Figure 7. Both are $10.5 \mathrm{~m}$ long. The width of the airplane is $6 \mathrm{~m}$. Figure 8 shows the target position in the moving coordinate system $\mathcal{R}^{\prime}$. The scattered fields of both targets in copolarizations and cross polarizations are computed from numerical code presented in [22]. We notice that the scattered fields of both targets could not be considered as a simple sum of point scatterers (isotropic or nonisotropic). Therefore, the first hypothesis of Section 2.2 is not ensured: the scattered fields of both targets vary as a function of the target position (indexed by $k T_{s}$ ). This configuration allows us to test the performance and the robustness of our MISAR processors in a realistic configuration in terms of frequencies, transmitter locations, integration time, and complex targets.

4.2. Study of the Impact of the Geometric Configuration on Image Resolutions. In this subsection, we focus on the final image resolution and sidelobe levels that could be obtained by the MIISAR processor in the configuration of Figure 5. The MIISAR processor is chosen because its result corresponds to the envelope of the MCISAR one. Therefore, the impact of parameters, such as the integration time and 


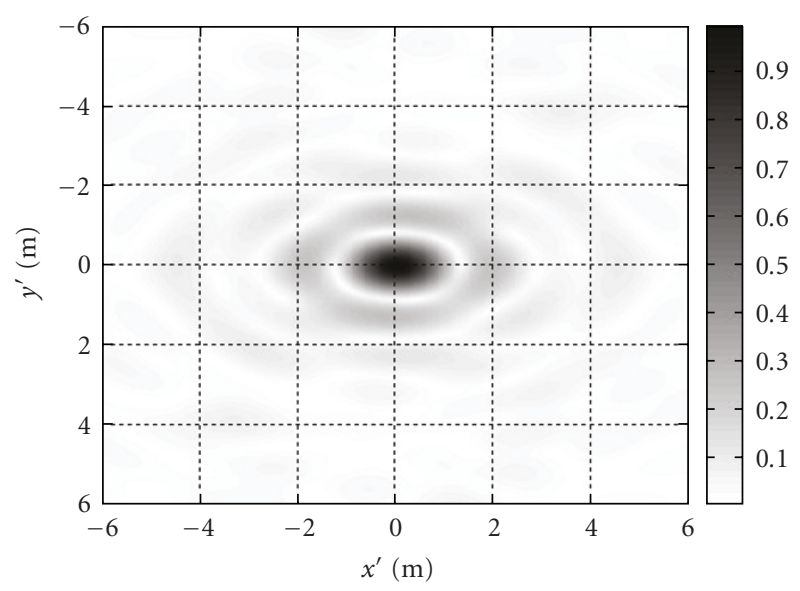

FIgURE 9: Reference image $I_{\text {ref }}$ of an isotropic point. SAL $=48 \mathrm{~km}$ and $N=103$ transmitters.

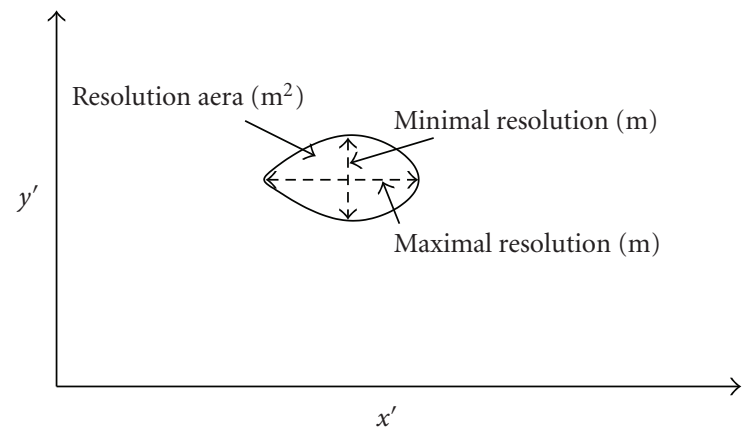

Figure 10: Definition of resolution parameters: maximal and minimal resolution and resolution aera.

the number of transmitters, on resolution and sidelobe levels is obviously the same for both processors.

Our resolution study is similar to the one reported in $[8,9]$. First, let the target be an isotropic point located at coordinate $(0,0)$. The result for this target and for a given configuration is obtained by the MIISAR processor. An ellipse is obtained from this image by computing the image level at the maximum minus $3 \mathrm{~dB}$. Several parameters, defined in Figure 10, are determined from this ellipse to measure the quality of the image resolution: the area of the ellipse in $\mathrm{m}^{2}$, and the maximal and minimal resolution in $m$ (the large and small axes of the ellipse).

To study sidelobe levels, the following parameter, denoted the Mean Absolute Error (MAE), is used:

MAE

$$
=10 \log _{10}\left(\frac{1}{M N} \sum_{i=1}^{N} \sum_{j=1}^{M}\left|I\left(x^{\prime}(i), y^{\prime}(j)\right)-I_{\mathrm{ref}}\left(x^{\prime}(i), y^{\prime}(j)\right)\right|\right),
$$

where $M$ and $N$ are the number of pixels of the image and $I_{\text {ref }}$ is a reference image, which is assumed of high quality in terms of resolution and sidelobe levels.

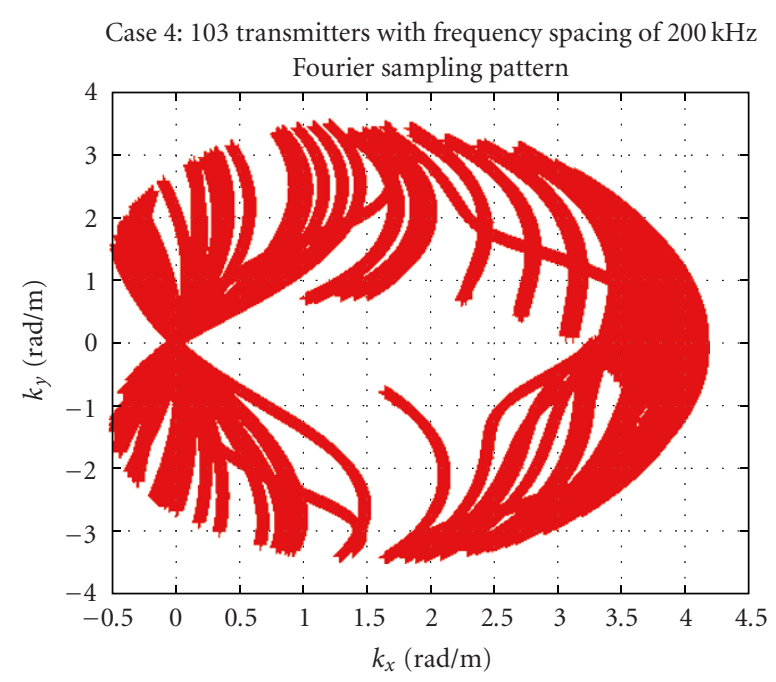

(a)

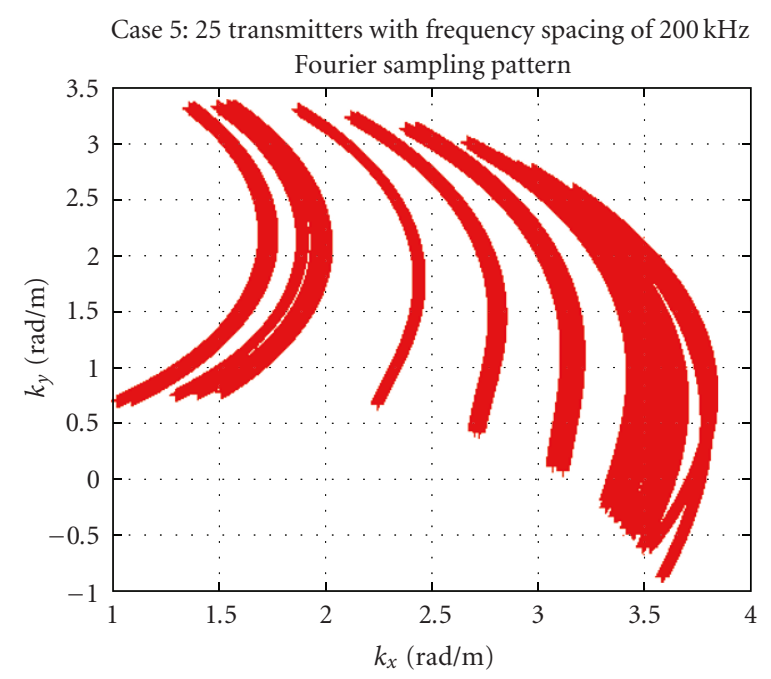

(b)

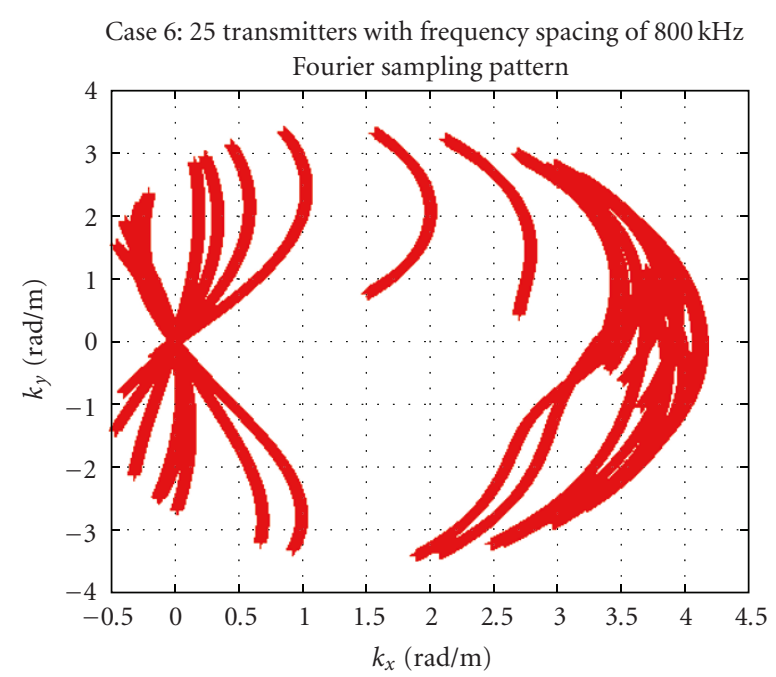

(c)

Figure 11: Fourier plan of MIISAR result for Case 4 (a), Case 5 (b), and Case 6 (c). 


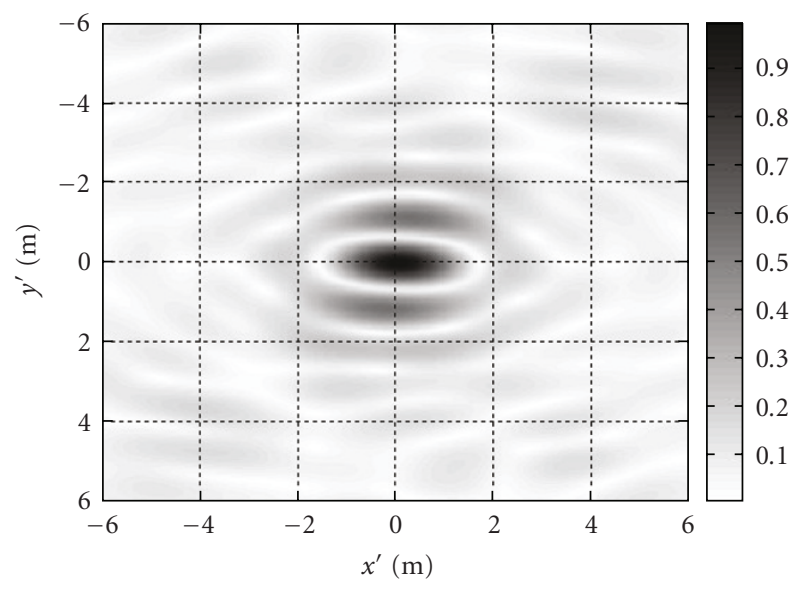

FIGURE 12: Image $I$ of an isotropic point in the chosen configuration. SAL $=32 \mathrm{~km}$ and $N=25$ transmitters with a frequency spacing of $800 \mathrm{kHz}$.

The reference image is obtained with a Synthetic Array Length (SAL) of $48 \mathrm{~km}$ and all 103 transmitters. This image is shown in Figure 9. Image resolution is quite good, with values of about $1 \mathrm{~m}$. Moreover, we believe that the sidelobe levels are sufficient for imaging of both targets of Figure 7.

Now, we describe all cases tested as a function of the $S A L$ and $N$ from the geometric configuration of Figure 5.

Case 1. $\mathrm{SAL}=4 \mathrm{~km}$ and $N=103$ transmitters.

Case 2. SAL $=8 \mathrm{~km}$ and $N=103$ transmitters.

Case 3. $\mathrm{SAL}=16 \mathrm{~km}$ and $N=103$ transmitters.

Case 4. SAL $=32 \mathrm{~km}$ and $N=103$ transmitters.

Case 5. SAL $=32 \mathrm{~km}$ and $N=25$ transmitters, with a frequency spacing of $200 \mathrm{kHz}$.

Case 6. $\mathrm{SAL}=32 \mathrm{~km}$ and $N=25$ transmitters, with a frequency spacing of $800 \mathrm{kHz}$.

Resulting image resolutions and MAE calculations (for the study of the sidelobe levels) are summarized in Table 2.

It is first to be noted that relatively good image resolution is obtained with a small SAL when all transmitters are considered. This might mislead us into thinking that a small SAL is sufficient to obtain usable images of complex targets. Unfortunately, the sidelobe levels are high for these cases, as indicated by the MAE value. The MAE decreases as the SAL increases, which is reasonable. In particular, the result for an SAL of $32 \mathrm{~km}$ and 103 transmitters seems promising in terms of resolution and sidelobe levels. However, we are interested in reducing the number of transmitters to reach a less complex radar system; also, it is unlikely that so many transmitters could be used in practice.

Therefore, let us consider less transmitters. To better comprehend the results of Cases 4-6 in Table 2, Figure 11 shows the corresponding Fourier coverage (see $[8,9]$ for more details) of the images obtained with the MIISAR processor. The best case, which best fills the Fourier plane, is reached when all of the 103 transmitters are considered. When only 25 transmitters with a frequency spacing of $200 \mathrm{kHz}$ are considered, the resolution performance is strongly degraded ( $1.2 \mathrm{~m}$ to $3 \mathrm{~m}$ for the maximal resolution). We notice that the Fourier plane does not cover the same area as in Case 4 (with 103 transmitters). In our geometric configuration, image resolution is influenced by the total frequency spacing provided by the considered transmitters. This value is identical in Cases 4 and $6(103 * 200 \mathrm{kHz}$ $\approx 25 * 800 \mathrm{kHz}$ ), and therefore the image resolutions are similar. Nevertheless, the MAE (linked to sidelobe levels) is slightly lower $(-30.8 \mathrm{~dB}$ against $-32.1 \mathrm{~dB})$ because the center of the Fourier plane is less filled in Case 6 than in Case 4. But, this value is more important in Case 6 than in Case 5 $(-30.8 \mathrm{~dB}$ against $-27.1 \mathrm{~dB})$.

To conclude, Case 6 provides results close the reference, but with lower complexity. Figure 12 shows the image of an isotropic point in this case. This image is close to the reference, shown in Figure 9. In the following, we choose this Radar configuration: SAL equal to $32 \mathrm{~km}$ and 25 transmitters with a frequency spacing of $800 \mathrm{kHz}$.

4.3. Simulation Results. In this subsection, we apply the MCISAR and MIISAR processors within the simulation context of Section 4.1. In this discussion, copolarization refers to $\theta \theta$ polarization and cross polarization refers to $\theta \phi$ polarization.

In a first simulation, we present bistatic images of the "cycone" and of the airplane obtained considering one transmitter and one receiver. For simulation, it is the transmitter operating at the highest center frequency that is chosen. The SAL is $32 \mathrm{~km}$. The result in copolarization for both targets is shown in Figure 13. From such results, the determination of the characteristics of the targets (length and width) is not trivial. Nevertheless, both images exhibit slight differences although the target shapes are similar. Indeed, energy in the airplane image is more concentrated. This allows us to expect interesting results in the multistatic context.

In a second simulation, the multistatic context is considered. The configuration that is taken is the one described in Section 4.2 for which the SAL is $32 \mathrm{~km}$ and 25 transmitters with a frequency spacing of $800 \mathrm{kHz}$ are used. Figure 14 shows the results of MCISAR and MIISAR processors for the "cycone" and for the airplane for copolarization. Both the processors enable to determine some target characteristics along the $y^{\prime}$-axis, such as the length (in the order of $10 \mathrm{~m}$ ) and the position of the back (located at $y^{\prime}=-9 \mathrm{~m}$ ). However, the width of the cylinder can only be measured with the MCISAR processor (in the order of $2 \mathrm{~m}$ ). The airplane and "cycone" images resulting from the MCISAR processor are similar. On the contrary, the airplane and the "cycone" images created by the MIISAR processor appear to be different; the energy maximum is concentrated in the back of the airplane and a wing seems visible at position $\left(x^{\prime}, y^{\prime}\right)=$ $(-4.5,6) \mathrm{m}$. Both processors seem capable of determining 


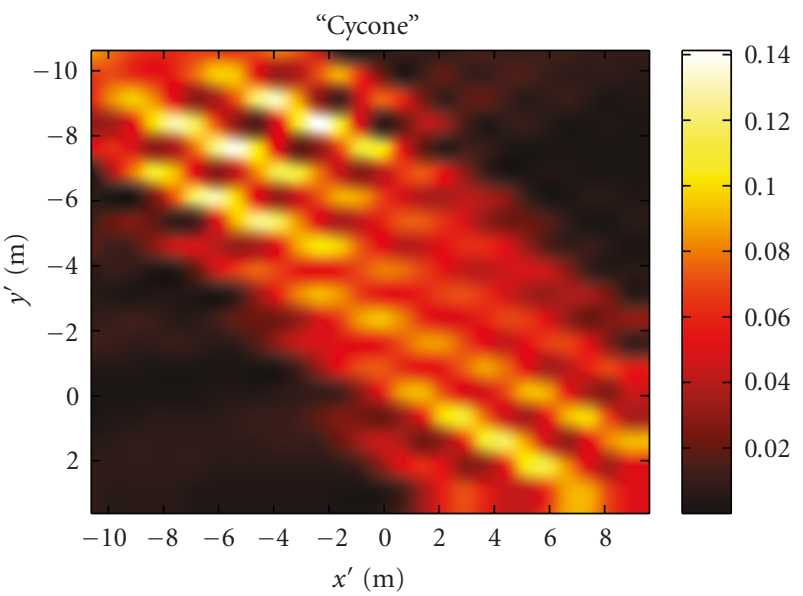

(a)

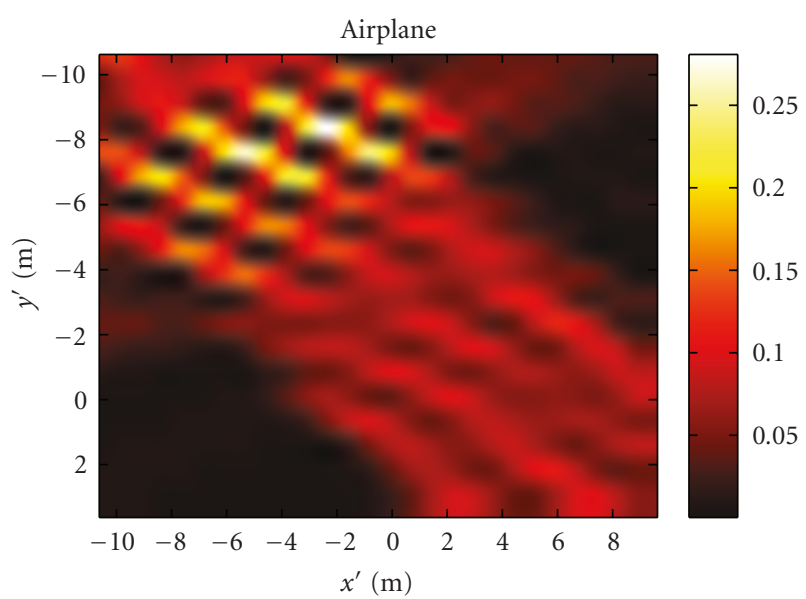

(b)

FIGURE 13: Bistatic image in copolarization of the "cycone" (a) and airplane (b).

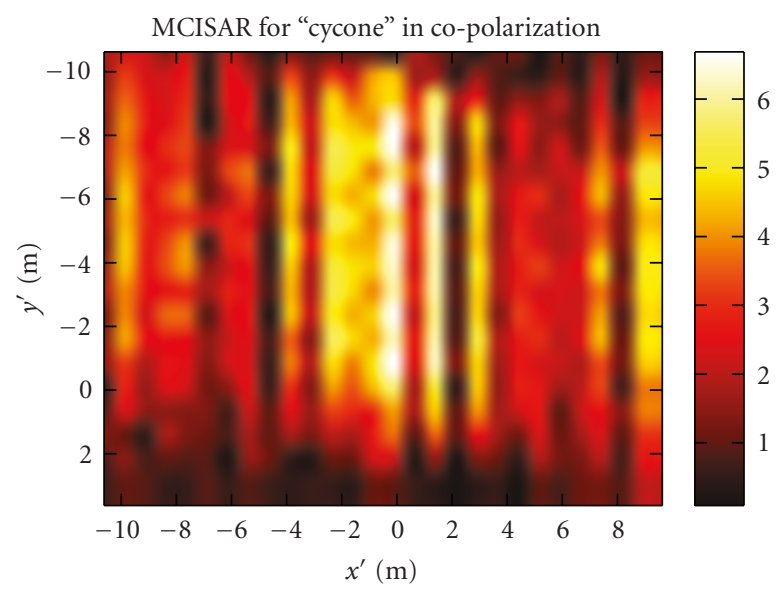

(a)

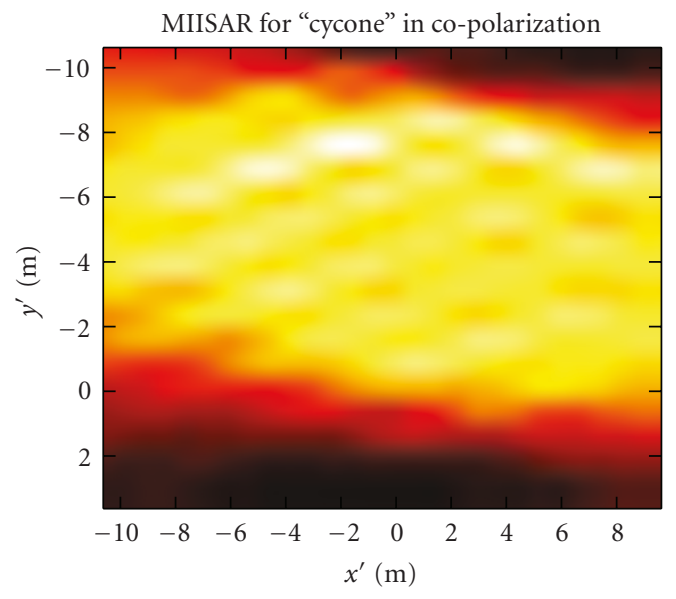

(c)

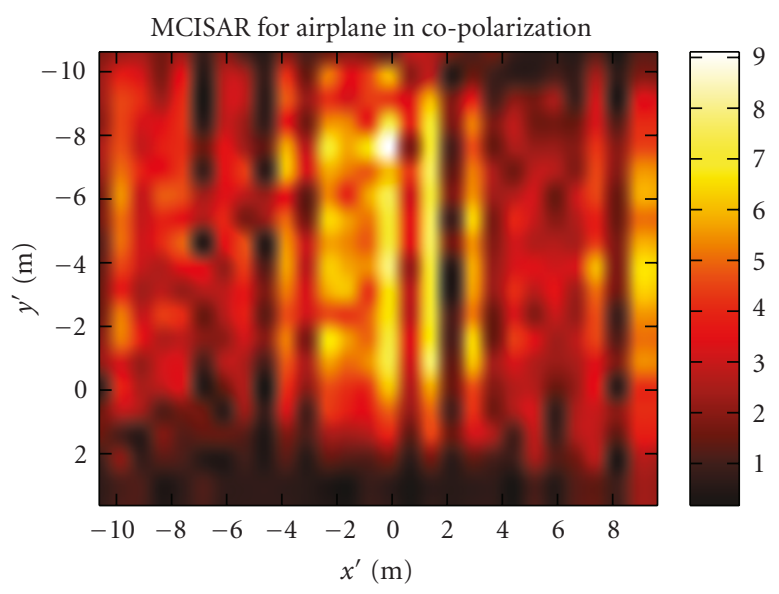

(b)

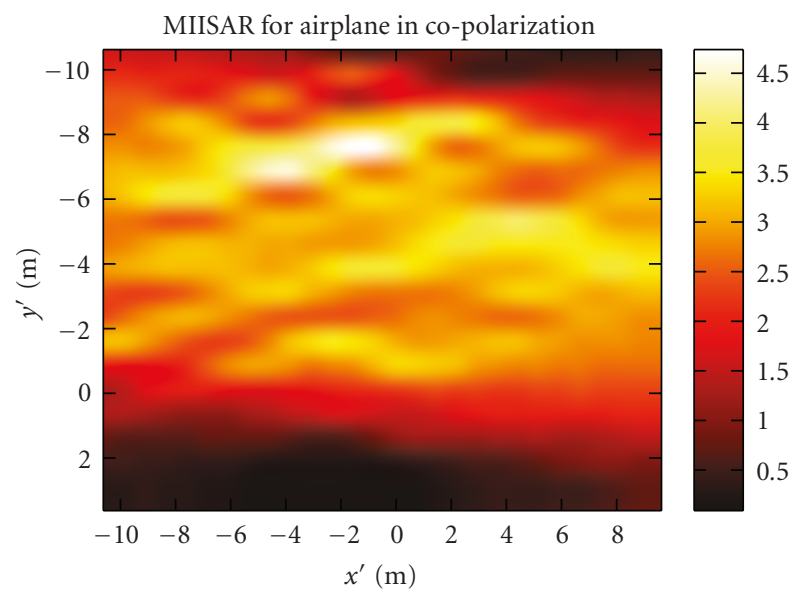

(d)

FIGURE 14: MCISAR results in copolarization of the "cycone" (a) and airplane (b) and MIISAR results in copolarization of the "cycone" (c) and airplane (d). 


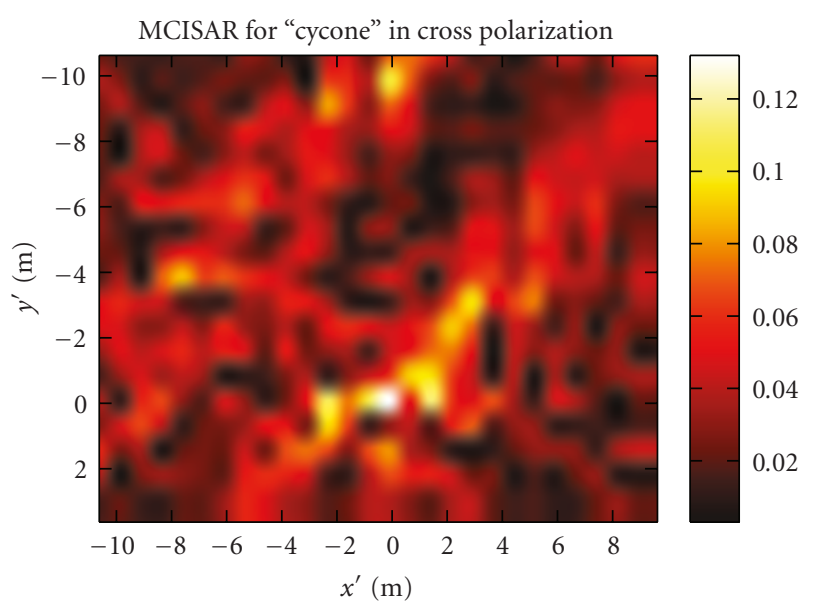

(a)

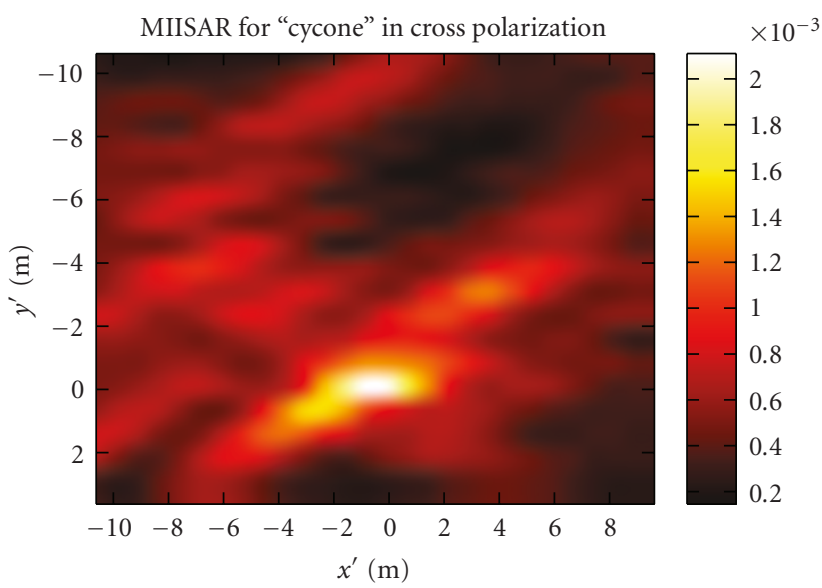

(c)

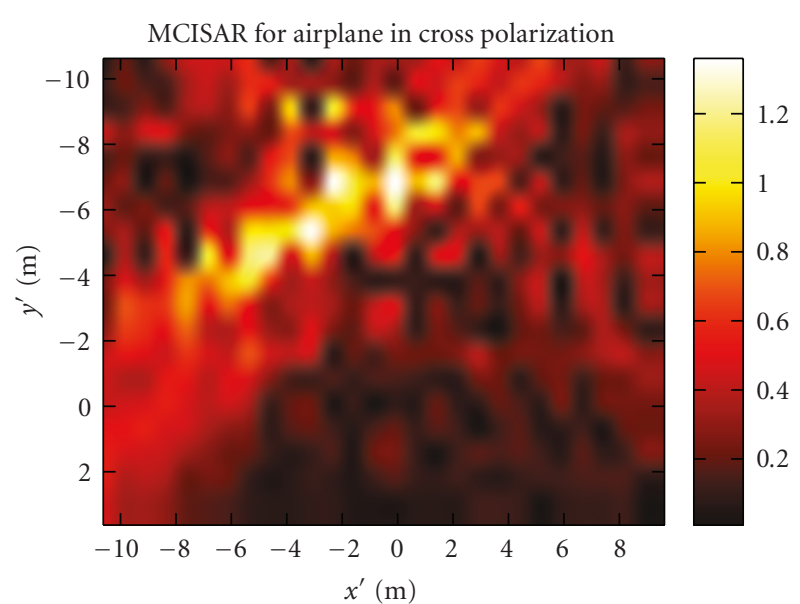

(b)

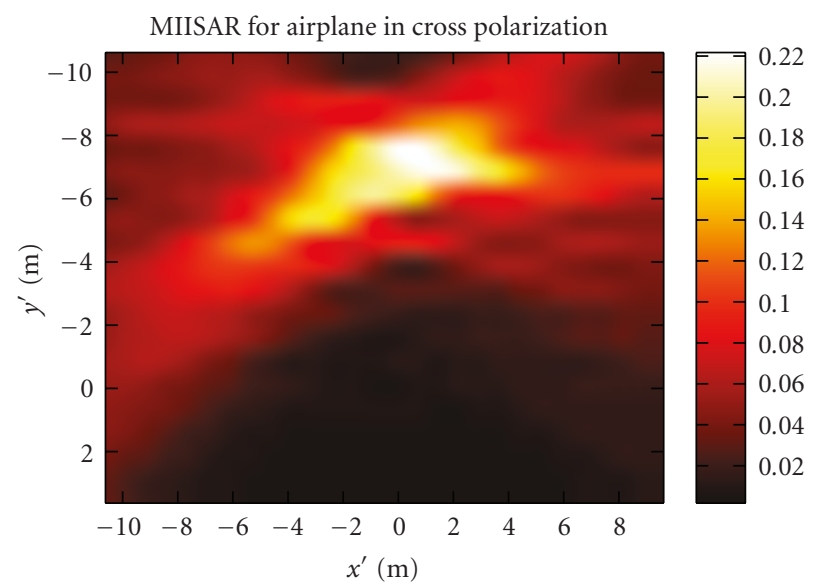

(d)

FIGURE 15: MCISAR results in cross polarization of the "cycone" (a) and airplane (b) and MIISAR results in cross polarization of the "cycone" (c) and airplane (d).

TABLE 2: Results of resolution parameters and MAE for Cases 1-6.

\begin{tabular}{|c|c|c|c|c|c|c|c|}
\hline Number of the case & 1 & 2 & 3 & 4 & 5 & 6 & ref \\
\hline SAL $(\mathrm{km})$ & 4 & 8 & 16 & 32 & 32 & 32 & 48 \\
\hline Number of transmitters $N$ & 103 & 103 & 103 & 103 & 25 & 25 & 103 \\
\hline Frequency Spacing $(\mathrm{kHz})$ & 200 & 200 & 200 & 200 & 200 & 800 & 200 \\
\hline Resolution Area $\left(m^{2}\right)$ & 0.96 & 0.91 & 0.86 & 0.84 & 2.82 & 0.72 & 0.73 \\
\hline Maximal resolution $(m)$ & 1.16 & 1.21 & 1.3 & 1.2 & 3 & 1.43 & 1.18 \\
\hline Minimal resolution $(m)$ & 1.05 & 0.95 & 0.84 & 0.89 & 1.22 & 0.64 & 0.78 \\
\hline $\operatorname{MAE}(\mathrm{dB})$ & -22.3 & -27.5 & -30.2 & -32.1 & -27.1 & -30.8 & $-\operatorname{Inf}$ \\
\hline
\end{tabular}

basic target parameters like length, width, and so forth, but only the MIISAR processor gives two images with enough difference to make the difference between the two targets.

Figure 15 shows results of MCISAR and MIISAR processors for the "cycone" and the airplane for cross-polarization. The energy in the airplane image is $20 \mathrm{~dB}$ higher than the energy for the "cycone" one. In particular, the energy in the "cycone" image is too weak to enable the results to be interpreted. For the airplane, the MIISAR result seems more interesting than the MCISAR result; the energy is higher and the phenomenon that creates the cross-polarization response is well localized near the back and the beginning of the wings.

These results show that both processors applied to copolarization and cross-polarization data might help developing classification rules. Moreover, the processors appear to be robust with respect to violations of the hypotheses they were derived under. 


\section{Conclusion}

This paper developed Multistatic ISAR processors from a detection theory viewpoint. From two hypotheses about the considered target, we developed two MISAR processors.

(i) The first processor, called MCISAR, is based on the isotropic point hypothesis. It is analogous to classical processors as it coherently sums bistatic images.

(ii) The second processor, called MIISAR, assumes that the scattered field of the target is different for each narrowband transmitter (realistic hypothesis in our context). This processor incoherently sums bistatic images.

Numerical simulations have been done in order to qualitatively illustrate the potential of the MIISAR processor in cases where the isotropic point hypothesis is not respected.

The last section applied the two considered processors to two similar complex targets in a realistic multistatic FM passive radar scenario. A study of the resolution and sidelobe levels has shown that it is not necessary to take every transmitters into account to get an optimal result. However, using transmitters that provide with wide frequency spacing and a large SAL is needed to achieve decent results.

Copolarization results from the MCISAR processor allowed us to determine some simple parameters of both the considered targets, such as the length and the width of the cylinder; however, images of both targets were similar and the wings and the cone were not seen. The MIISAR processor is proved to be more performant for discriminating the two targets because the images provided in copolarization are different. Moreover, the cross-polarization images obtained by the MIISAR processor exhibited many differences. According to those results, the proposed imaging algorithms may be used as first steps of a classification process using multifrequency and multistatic passive radar.

Future work will deal with more complex target models and the development of associated imaging algorithms based on the monostatic SAR processors presented in $[19,20]$ where subspace approaches are used to integrate complex target model in monostatic SAR processing.

\section{References}

[1] H. Cantalloube and P. Dubois-Fernandez, "Airborne x-band sar imaging with $10 \mathrm{~cm}$ resolution: technical challenge and preliminary results," in Proceedings of the 5th European Conference on Synthetic Aperture Radar (EUSAR '04), Ulm, Germany, May 2004.

[2] P. E. Howland, D. Maksimiuk, and G. Reitsma, "FM radio based bistatic radar," IEE Proceedings: Radar, Sonar and Navigation, vol. 152, no. 3, pp. 107-115, 2005.

[3] P. E. Howland, "Target tracking using television-based bistatic radar," IEE Proceedings: Radar, Sonar and Navigation, vol. 146, no. 3, pp. 166-174, 1999.

[4] L. Ehrman and A. D. Lanterman, "Automated target recognition using passive radar and coordinated flight models," in Automatic Target Recognition XIII, F. A. Sadjadi, Ed., vol. 5094 of Proceedings of SPIE, pp. 196-207, Orlando, Fla, USA, April 2003.
[5] L. M. Ehrman and A. D. Lanterman, "A robust algorithm for automatic target recognition using passive radar," in Proceedings of the 36th IEEE Annual Southeastern Symposium on System Theory, pp. 102-106, Atlanta, Ga, USA, March 2004.

[6] L. M. Ehrman and A. D. Lanterman, "A robust algorithm for automated target recognition using precomputed radar cross sections," in Automatic Target Recognition XIV, F. A. Sadjadi, Ed., vol. 5426 of Proceedings of SPIE, pp. 197-208, Orlando, Fla, USA, April 2004.

[7] S. M. Herman, A particle filtering approach to joint passive radar tracking and target classification, Ph.D. thesis, University of Illinois, Urbana, Ill, USA, 2002.

[8] F. Maussang, F. Daout, G. Ginolhac, and F. Schmitt, "Multistatic scenarios for a GPS SAR system," in Proceedings of the International Geoscience and Remote Sensing Symposium (IGARSS '08), vol. 3, pp. 270-273, Boston, Mass, USA, July 2008.

[9] F. Daout, F. Schmitt, and G. Ginolhac, "Performance analysis of a bistatic/multistatic passive sar system based on a GPS signal," submitted to IEEE Transactions on Geoscience and Remote Sensing.

[10] M. Soumekh, Synthetic Aperture Radar Signal Processing, Wiley-Interscience, New York, NY, USA, 1999.

[11] D. L. Mensa and G. R. Heidbreder, "Bistatic synthetic-aperture radar imaging of rotating objects," IEEE Transactions on Aerospace and Electronic Systems, vol. 18, no. 4, pp. 423-431, 1982.

[12] J. L. Walker, "Range-doppler imaging of rotating objects," IEEE Transactions on Aerospace and Electronic Systems, vol. 16, no. 1, pp. 23-52, 1980.

[13] Y. Wu and D. C. Munson Jr., "Multistatic synthetic aperture imaging of aircraft using reflected television signals," in Algorithms for Synthetic Aperture Radar Imagery VIII, E. G. Zelnio, Ed., vol. 4382 of Proceedings of SPIE, pp. 1-12, Orlando, Fla, USA, April 2001.

[14] Y. Wu and D. C. Munson Jr., "Wide-angle ISAR passive imaging using Smoothed Pseudo Wigner-Ville distribution," in Proceedings of the IEEE National Radar Conference, pp. 363368, Atlanta, Ga, USA, May 2001.

[15] Y. Wu and D. C. Munson Jr., "Multistatic passive radar imaging using the Smoothed Pseudo Wigner-Ville distribution," in Proceedings of IEEE International Conference on Image Processing (ICIP '01), vol. 3, pp. 604-607, Thessaloniki, Greece, October 2001.

[16] A. D. Lanterman, D. C. Munson Jr., and Y. Wu, "Wideangle radar imaging using time-frequency distributions," IEE Proceedings: Radar, Sonar and Navigation, vol. 150, no. 4, pp. 203-211, 2003.

[17] A. D. Lanterman and D. C. Munson Jr., "Deconvolution techniques for passive radar imaging," in Algorithms for Synthetic Aperture Radar Imagery IX, E. G. Zelnio, Ed., vol. 4727 of Proceedings of SPIE, pp. 166-177, Orlando, Fla, USA, April 2002.

[18] M. Cetin and A. D. Lanterman, "Region-enhanced passive radar imaging," IEE Proceedings: Radar, Sonar and Navigation, vol. 152, no. 3, pp. 185-194, 2005.

[19] R. Durand, G. Ginolhac, L. Thirion, and P. Forster, "New SAR processor based on matched subspace detectors," IEEE Transactions on Aerospace and Electronic Systems, vol. 45, no. 1, pp. 221-236, 2009.

[20] R. Durand, G. Ginolhac, L. Thirion, and P. Forster, "SAR processor based on a CFAR signal or interference subspace detector matched to man made target detection in a forest," in Proceedings of IEEE International Conference on Acoustics, 
Speech, and Signal Processing (ICASSP'07), vol. 2, pp. 293-296, Honolulu, Hawaii, USA, April 2007.

[21] Agence National des Fréquences, "Emplacement des émetteurs fm en france," http://www.cartoradio.fr/netenmap.php?cmd= zoomfull.

[22] F. Daout, F. Schmitt, and G. Ginolhac, "Computation of bistatic ser with nec2 in a context of passive isar system," in Proceedings of IEEE Geoscience and Remote Sensing Symposium (IGARSS '05), Seoul, South Korea, July 2005.

[23] M. N. Petsios, E. G. Alivizatos, and N. K. Uzunoglu, "Manoeuvring target tracking using multiple bistatic range and rangerate measurements," Signal Processing, vol. 87, no. 4, pp. 665686, 2007.

[24] P. Forster and M. Lesturgie, "Passive radar for target tracking and classification," in Proceedings of the International Radar Conference, Toulouse, France, October 2004.

[25] M. Tobias and A. D. Lanterman, "Probability hypothesis density-based multitarget tracking with bistatic range and Doppler observations," IEE Proceedings: Radar, Sonar and Navigation, vol. 152, no. 3, pp. 195-205, 2005.

[26] M. Tobias and A. D. Lanterman, "Techniques for birth-particle placement in the probability hypothesis density particle filter applied to passive radar," IET Radar, Sonar and Navigation, vol. 2, no. 5, pp. 351-365, 2008.

[27] B. D. Rigling and R. L. Moses, "Polar format algorithm for bistatic SAR," IEEE Transactions on Aerospace and Electronic Systems, vol. 40, no. 4, pp. 1147-1159, 2004. 